\title{
Patient Perspective on Watchful Waiting/Active Surveillance for Localized Prostate Cancer
}

\author{
Jinping Xu, MD, MS, Anne Victoria Neale, PhD, MPH, Rhonda K. Dailey, MD, \\ Susan Eggly, PhD, and Kendra L. Schwartz, MD, MSPH
}

Objective: To describe prostate cancer treatment decision making, focusing on knowledge and attitudes toward observation, known as watchful waiting (WW) or active surveillance (AS), and reasons for not choosing WW/AS.

Methods: Semistructured in-person interviews were conducted with 21 men (14 black; 7 white) with recently diagnosed localized prostate cancer.

Results: All cancers were detected by prostate-specific antigen screening; 14 men had low-risk disease. Nineteen chose surgery or radiation treatment. The majority wanted to "get rid of" or "cure" the cancer by undergoing aggressive therapy, even with awareness of the potential for significant side effects. Most men seemed unaware of the uncertainty/controversies that aggressive treatment may not cure their cancer or improve their survival. Limited knowledge about WW/AS was common, and few remembered WW/AS being presented as a viable option. Rather, many men perceived it as "doing nothing." Some men, who initially were inclined toward WW/AS, yielded to pressure from family, physicians, or both to choose aggressive treatment. Lack of physician support was a significant barrier to WW/AS.

Conclusions: The observational strategy (WW/AS) was not viewed as a reasonable approach, even for those with low-risk cancer. The desire for aggressive therapy may reflect the complex psychology associated with receiving a diagnosis of cancer and the limited supportive counseling received. Further efforts to better understand and educate patients and physicians may help men make informed and appropriate treatment decisions to maximize quality of life without compromising survival. ( $\mathrm{J}$ Am Board Fam Med 2012;25:763-770.)

Keywords: Active Surveillance, Decision Making, Medical Oncology, Prostate Cancer, Watchful Waiting

Widespread use of prostate-specific antigen (PSA) screening in the United States has led to increased diagnoses of early stage prostate cancer in an ever younger group of men. ${ }^{1}$ Ninety percent of cancers detected by PSA screening are localized prostate cancers (LPCs); more than half

This article was externally peer reviewed.

Submitted 16 May 2012; revised 1 August 2012; accepted 6 August 2012.

From the Department of Family Medicine and Public Health Sciences (JX, AVN, RKD, KLS); and the Behavioral Oncology and Communication Program (SE), Karmanos Cancer Institute, Wayne State University, Detroit, MI.

Funding: This study is funded by American Cancer Society (grant number: MRSGT-06-133-01-CPPB).

Prior presentation: The abstract was presented at the 37 th Annual Meeting of the North American Primary Care Research Group (NAPCRG), Montreal, Quebec, Canada, November 14-18, 2009.

Conflict of interest: none declared.

Corresponding author: Jinping Xu, MD, MS, 3939 Woodward Ave, 2nd Floor, Detroit, MI 48021 (E-mail: jxu@med.wayne.edu). are low-risk, small-volume tumors unlikely to become life threatening. Aggressive treatment (surgery or radiation) may provide little survival benefit for such men, whereas increasing the risk of side effects that can severely impact quality of life. ${ }^{2-11}$ Nevertheless, approximately $90 \%$ of patients, even those with low-risk disease, undergo aggressive treatment, ${ }^{12-14}$ and many then experience short- and long-term side effects such as impotence and incontinence. From 1986 to 2005, an estimated one million American men who would not otherwise have been treated received surgery, radiation therapy, or both after positive PSA screening. Of those, 5,000 died from complications and an estimated 300,000 developed impotence, incontinence, or both. ${ }^{1}$

The long natural history of prostate cancer suggests that a strategy of initial observation/surveillance with selected intervention would approximate 
the results achieved by aggressive treatment of all low-risk patients at the time of diagnosis. ${ }^{15,16}$ The concept of observational strategy, mostly known as watchful waiting $(W W)^{17-19}$ but more recently called active surveillance (AS), ${ }^{7,16,20}$ offers the opportunity to delay aggressive treatment and its associated morbidities until cancer progresses. ${ }^{17-20}$ Until recently, WW usually meant waiting to start treatment until the cancer caused symptoms. Recently, frequent monitoring is more common and curative treatment is recommended if early evidence of cancer progression is found. Some physicians still consider this to be $W W,{ }^{17,18}$ whereas others consider this to be different from WW and call it active surveillance (AS). ${ }^{20,21}$

Because the impact of prostate cancer treatment options on patients' physical and psychological well-being varies, and because treatment outcomes are uncertain in the individual case, practice guidelines in the United States recommend shared decision making between clinician and patient. ${ }^{7,21}$ Little is known about what leads men to select aggressive treatment, and we know even less about why WW/AS is not a serious consideration when deciding upon the initial treatment. ${ }^{22,23}$ Historically, black men undergo less aggressive treatment and more WW, even after adjustment for socioeconomic status and comorbidities. $^{24-27}$ These older studies often were unable to determine whether men were being observed, closely monitored, or simply not treated. Black men who were managed with WW often had inadequate monitoring, which likely contributed to poorer outcome. ${ }^{28}$ Black men also reported more decision-making difficulty, distress, and decision regret. ${ }^{29-32}$ The reasons for these racial differences remain unclear.

To better understand the LPC treatment decision-making process, its influencing factors, and any racial/cultural differences, we conducted a qualitative study of black and white men with LPC detected with a PSA screen. In this article, we focus on men's self-reported knowledge and attitudes about WW/AS as an initial management strategy. Understanding the reason(s) for not choosing WW/AS is the critical first step to improve appropriate uptake of WW/AS by men with low-risk disease. We used WW and AS synonymously as WW/AS unless otherwise specified.

\section{Methods}

Data reported here are from a semistructured, inperson interview study that explored factors influencing the initial treatment selection by men with recently diagnosed localized prostate cancer. Details about the sampling strategy, data collection, and analysis already have been published. ${ }^{33}$ Briefly, we interviewed a purposive sample of 21 men (14 black, 7 white) with LPC who were recruited from offices of urologists and radiation oncologists in metropolitan Detroit. Before the study began, we reviewed the relevant literature and then constructed our conceptual model ${ }^{33}$ on the basis of behavioral theories. ${ }^{34,35}$ Under the guidance of this model, we then developed the interview guide (available from the authors upon request). Openended questions were followed by more detailed prompts about the factors influencing treatment selection and the men's beliefs about the efficacy and side effects of each treatment option. Interviewers probed for understanding of the advantages and disadvantages of surgery, radiation, and WW/ AS, respectively. We used the theoretical preconceptions from our model to build a preliminary skeleton, or axis, of codes, and 3 authors (JX, RD, and SE) independently read the transcripts to look for recurring emergent themes using an immersion/crystallization analysis technique. ${ }^{36,37}$ After agreement on the relevant themes, we developed operational definitions of the themes. Each transcript then was coded independently by at least 2 reviewers and compared using Atlas.ti qualitative data analysis software. Consensus was reached by discussion. The main findings were presented to 10 interviewees for their feedback and confirmation of the findings.

\section{Results}

\section{Study Sample}

Twenty of the 21 men with LPC were interviewed within 6 months of the initial diagnosis; one man who chose WW/AS had been diagnosed about 2 years earlier. Collectively, these men consulted 12 urologists and 6 radiation oncologists in the Detroit metropolitan area. Fourteen had low-risk prostate cancers (ie, Gleason score $\leq 6$, PSA level $<10 \mathrm{ng} / \mathrm{mL}$, and clinical staging $\mathrm{T} 1$ and $\mathrm{T} 2$ ); all chose aggressive treatment (either surgery or radiation). Two men with a Gleason score of 7 and a PSA level $<10 \mathrm{ng} / \mathrm{mL}$ were characterized as having 
"intermediate-risk" disease; both chose radiation. Five men with incomplete tumor data (missing PSA level, Gleason score, or both) were characterized as having "unknown-risk" disease; 2 chose surgery, 1 cryotherapy, and 2 chose WW/AS. ${ }^{33}$ In this article, we focus on the comments made about the WW/AS approach and the reasons for not choosing WW/AS. If men had never heard of WW/AS, we explained it as "close watching/monitoring of the cancer but not giving immediate treatment unless there are changes suggesting cancer growth, with the intent to avoid problems/side effects that may be caused by treatment." Men then were asked to describe what they would do if they had known about WW/AS while deciding on a treatment.

\section{Knowledge of WW/AS}

Eighteen men were aware of WW/AS as potential treatment option but had limited knowledge and many misunderstandings. Most remembered their urologist presenting several treatment options, but only half recalled that the WW/AS option was mentioned. Some learned about WW/AS by reading the brochure provided by their urologists or via the Internet. Few remembered WW/AS presented as a serious alternative; usually it was mentioned to explain its inappropriateness and to justify the preferred aggressive treatment. For example,

"He [urologist] mentioned doing nothing [WW/AS]. It's funny, everybody mentioned that, but then everyone said, I wouldn't recommend it for you." (white man, 59 years old, radiation, intermediate risk)

"Well, the doctor never brought it [WW/AS] up to me." (black man, 55 years old, radiation, low risk)

\section{Attitudes and Beliefs About WW/AS}

Most men believed cancer should be treated and, better yet, cured as soon as possible because of the belief that any cancer, even slow-growing prostate cancer, would grow, spread, and kill if not treated. One man who did not know about WW/AS before our interview said that it "would have scared me to death." Compared with aggressive treatment such as surgery or radiation, WW/AS was consistently referred to as "doing nothing," and many men attributed this phrase to urologists.

"Doctor said, 'you could do nothing, but I think you could have a problem later."”

"If you watch and do nothing, it [cancer] is going to obviously progress. It's like, well, you're waiting for the time bomb to explode." (black man, 57 years old, radiation, low risk)

"I'm trying to think bow he [urologist] described it. ... I may not even have paid attention to it because when he mentioned it [WW/AS], it was like, 'No, we are not going to do that'. I ruled that out immediately." (white man, 49 years old, surgery, low risk)

Some men thought WW/AS equaled "burying your head in the sand" or "giving up."

"If you want to live, you just have to make decisions to do things to extend your life if you can. But if you want to give up, you can give up or just wait and see." (white man, 59 years old, radiation, intermediate risk)

Although some men understood the rationale of WW/AS as an alternative to an aggressive treatment with potential side effects, they still considered it too risky and were afraid their treatment choices might be limited if cancer progressed. They worried their cancer might behave aggressively despite reassurance of a good prognosis because "cancer will spread and eventually kills you."

"I agree that I might die from something else, I just didn't want to roll the dice for me on that." (white man, 68 years old, radiation, low risk)

"It [WW/AS] seems logical, but I'm not doing it. I want to get rid of this thing [cancer] right now. I didn't want to give it a chance to do notbing. You've got to crush that bug. ... You've got cancer, man, you don't know what that thing's going to do." (black man, 62 years old, cryotherapy, low risk)

\section{Reasons for Rejecting WW/AS Relative "Youth"}

All men mentioned that their age (range, 48-70 years) was influential in their treatment decision. Being "young" was a justification for rejecting WW/AS because "WW/AS is only appropriate for older men." Men often described their current general health as good or excellent and used familial longevity to predict their own long life, another justification for rejecting WW/AS. Most men mentioned that their urologists explained WW/AS was only for older men who cannot have surgery, and these men felt lucky to be considered candidates for surgery. One man even commented that it would be "irresponsible" or even "ridiculous" for his doctors to suggest WW/AS for him at his age (53 years).

"It all comes down to the age question. I'm 60 years old and in good health. If I was 75 years old, it would be 
different." (white man, 60 years old, surgery, low risk)

"At the time I was 51 years old; just get it [cancer] out of me. If I was 80, 70, maybe even 60 years [old], I probably would say, wait and see." (black man, 51 years old, surgery, unknown risk)

"Well, you wouldn't do that [WW] for somebody my age. You'd only do it for older people. So $[W W]$ wasn't even on the table for me, and nobody suggested it. It absolutely wasn't even brought up! And if it had been, I would have laughed. I would have walked out of the office because I'd have felt this person doesn't know what he's talking about." (white man, 53 years old, radiation, low risk)

\section{"Doing Nothing” Is Not an Option}

Most men chose aggressive treatment to "get rid of cancer" or to be "cured" so they could move on with their life. Men generally were optimistic that some form of early treatment would lead to cure. Few men seemed aware that treatment might not improve their survival. "Doing nothing" was not an option for them, and the "watch and wait" approach defeated the whole purpose of getting regular health check-ups to "catch things early" enough to deal with them effectively.

"I have to do some sort of treatment ... something has got to be done." (white man, 49 years old, surgery, low risk)

"I knew I wasn't going to sit around and wait. I was going to make one of those choices: surgery or radiation. Or if I needed to have both." (black man, 51 years old, surgery, unknown risk)

"I'm interested in turning this thing [around]and getting on with my life. I don't want to think about it. So something that doesn't result in a cure is not viable to my mind." (white man, 60 years old, surgery, low risk)

Besides conventional cancer treatment such as surgery or radiation, most men talked about using complementary/alternative strategies to help them "fight" the tumor and gain some sense of control over their cancer. These included eating more vegetables, regular exercise, stress reduction, a positive attitude, vitamins, and other supplements.

\section{Anxiety and Fear of Cancer Spreading}

Fear of cancer progression caused many to reject WW/AS as "too risky." They mentioned familiarity with how cancer had affected others and feared the consequences of delaying treatment. Some expressed anxiety that cancer might spread during the time between diagnosis and treatment and pushed their doctors to move quickly. These men were reluctant to wait because cancer could be "spreading silently" even if it was "not growing fast now." Untreated cancer was like living with a "ticking time bomb." A few men commented that they chose an aggressive treatment to avoid future regret if the cancer became incurable.

"I don't want to leave it [cancer] alone because it might spread. I want to get it out of me if I can." (black man, 55 years old, radiation, low risk)

"I just want it [surgery] done as soon as possible because I knew what cancer could do, so I didn't want to take a chance. I just couldn't deal with it ... I know it's [cancer] in my body. It just seems like I was imaging things then. Before [my diagnosis], I didn't bave any symptoms. But between diagnosis and the surgery, it was like, 'oh, everything, is that the cancer?' So I'm just thinking, wow, if I had to go a year like this, no way!" (black man, 57 years old, surgery, low risk)

"Why would I wait when I can treat it and try to eradicate it now as to letting it progress possibly out of control?" (black man, 57 years old, radiation, low risk)

Men who perceived their cancer as more "serious" felt WW/AS was not a viable option. Although 14 men met criteria for a low-risk cancer diagnosis, half believed their cancer was "serious" and "could spread to other parts of the body" if not treated immediately. Other factors associated with the preferences for immediate treatment included having a family member die of prostate cancer, a perceived high Gleason score or PSA level, or tumor size.

"It [WW/AS] is not an option, because I have a seven [Gleason score]. That kind of cemented it that you don't do nothing. The seven to me was more serious." (white man, 59 years old, radiation, intermediate risk)

"I kind of thought about it [WW/AS], but then the surgeon suggested that there's a risk with WW/AS. The risk is I have it [cancer] in my body until it metastasizes. ... And then he said it was a large portion of my prostate, not just like in one little spot ... I don't want to take the risk of waiting around until it gets worse." (black man, 60 years old, surgery, low risk) 


\section{Persuasion Against WW/AS}

Many men said that their physicians (both urologists and radiation oncologists) thought WW/AS was not suitable for them, and they felt pressure to seek aggressive treatment. Five men (aged 53-70 years) seriously considered WW/AS, including one who called it "active surveillance." Avoiding potential side effects from aggressive treatment was the motivation for considering WW/AS. However, 3 of these 5 men ultimately chose radiation instead. One man (53 years old, black) had great concerns about the side effects of aggressive treatment, particularly impotence, and correctly understood that WW/AS meant that his cancer would be closely monitored. When the patient proposed WW/AS, he reported that his urologist "frowned on" the idea, stressing the hazards of delaying treatment. A university professor (68 years old, white) consulted 8 different physicians and was dissuaded from an initial decision for AS. The patient was satisfied and ready to take the advice of the last urologist he consulted from a major university who "said very decisively, 'if it were me, I would have active surveillance." However, the man's radiation oncologist changed his mind, stating that he was not a good candidate for AS because of his short PSA doubling time. The physician gave the patient a pertinent research article and he was subsequently convinced WW/AS was not the correct path for him "according to the latest research." The third man changed his mind because of fear of cancer; he stated, "I have seen [what] cancer does to people ... It's a long ride."

"So my thinking was, well, why can't we do the watch and wait because you can check it periodically. I proposed that [WW/AS] to [the urologist]and he sort of frowned on it and gave me reasons why not." (black man, 53 years old, radiation, low risk)

Men also described that their family members urged aggressive treatment. One initially preferred WW/AS plus nutrition supplements to avoid treatment-related side effects. However, his family pressured him to choose surgery and, as a compromise, he finally chose radiation. Another man stated he felt unspoken pressure from his family to choose aggressive treatment.

"My wife keeps asking me why I haven't chosen a treatment yet!" (white man, 53 years old, radiation, low risk)
"I think my family would be very upset if I took the wait and see approach." (white man, 68 years old, radiation, low risk)

\section{Discussion}

Based on current evidence that PSA screening causes more harm than benefit, primarily due to overdetection and overtreatment, the US Preventive Services Task Force recently released the draft of a new practice guideline indicating that healthy asymptomatic men should not receive routine PSA screening. ${ }^{38}$ This new guideline sparked intense media coverage and a national debate on medical decision making and the benefit of AS as an appropriate management strategy for men with low-risk prostate cancer. ${ }^{39}$ Overdetection causes patient anxiety and most often has led to additional evaluation and use of resources. In addition, the diagnosis of asymptomatic LPC is uniformly followed by treatment.

Decreasing overtreatment and its associated morbidity among men with low-risk LPC is an important public health issue given the large number of men affected and the risk for adverse outcomes, as recognized by a recent independent panel convened by the National Institutes of Health. ${ }^{41}$ The panel concluded that many men with low-risk prostate cancer should be closely monitored, permitting treatment-with its side effects- to be delayed until disease progression (AS). Annually, more than 100,000 men diagnosed with prostate cancer in the United States are candidates for this approach. ${ }^{41}$ However, observation strategy (WW/ AS) is not an intuitive pathway for patients to accept after receiving a cancer diagnosis. In our study, most men seemed unaware of the uncertainty or controversies that aggressive treatments may not "cure" their cancer or improve their survival.

Our findings support results from other qualitative studies that found that men with prostate cancer perceived WW/AS as "doing nothing" 42 and that there was a lack of physician support for WW/ AS. $^{22}$ Studies suggest men rely heavily on physician recommendations about their choice of treatment. ${ }^{22,42}$ Holmboe and Concato, ${ }^{23}$ however, reported the most frequent reason (64\% of 102 men) for not choosing WW was the patient's need to "do something" to "combat" their cancer. Physician recommendation was another, but much less frequent $(12 \%)$, reason to reject $W W$; however, $58 \%$ 
(7 of 12) of men who chose WW cited a physician recommendation as a reason for the choice. ${ }^{23}$ Two recent studies ${ }^{43,44}$ of men enrolled in an AS protocol found physician influence to be the greatest contributor to their decision to enroll.

Despite some subtle racial/cultural differences, mostly in trust of physicians and the medical system, we did not find any overt racial/cultural differences in men's treatment decision making. ${ }^{33}$ Demark-Wahnefried et al ${ }^{45}$ surveyed 231 men (50\% black) and found that physician recommendation was the most influential factor in deciding on a treatment for both blacks and whites and reported no differences between blacks and whites in WW discussion during clinical encounters. ${ }^{45}$ Some urologists may be reluctant to offer WW/AS to black men because black race may be an adverse prognostic factor for outcome, ${ }^{46,47}$ although others have refuted this, ${ }^{48}$ particularly for low-risk disease. ${ }^{49}$ We found that both black and white men considered the treatment choice to be theirs to make, with concern of cancer progression far surpassing the concerns of treatment side effects. Because of the explorative nature of our study, future research powered to identify any black/white differences is needed to clarify underlying factors in prostate cancer treatment decision making.

Although the US academic medical community and professional societies have become more accepting of AS for low-risk prostate cancer, ${ }^{7,9,21}$ delaying aggressive treatment still is not generally acceptable to most patients or their doctors. In a large community study, only $9.2 \%$ of men with low-risk cancer accepted AS. ${ }^{14}$ In contrast, a recent UK study showed British men and doctors were more willing to accept AS, with up to $39 \%$ opting for AS in recent years. ${ }^{50}$ One of the possible explanations for this is that the American culture values "doers," compounded by the "aggressiveness" of American medicine. ${ }^{51}$ This may be compounded further by the success of early detection campaigns. After decades of public messages about the "war on cancer" and "early detection and early treatment saves lives" in the United States, ${ }^{52}$ when informed that one has cancer, a "war" mentality may be "switched on" to do something to "fight it" or, better yet, "get rid of it" or "cure it" by whatever means as soon as possible. The warlike mentality may very well deprive men of the opportunity to live well and long with his cancer, both of which are realistic possibilities because virtually $100 \%$ of pa- tients with LPC detected by a PSA screen survive $\geq 5$ years and more than $90 \%$ survive 10 years. ${ }^{11,53}$ For AS to become a more prevalently adopted treatment, health care professionals will have to address the perception of AS as the "doing nothing" approach. The fear and anxiety after a cancer diagnosis may overwhelm any discussion about some prostate cancers that may best be managed initially with a surveillance strategy.

\section{Strengths and Weaknesses of the Study}

To our knowledge, this study is one of the first to report on black men's experiences and perspectives regarding WW/AS, about which little information has existed to date. We included participants with a wide range of education and socioeconomic backgrounds who consulted multiple specialists, including both urologists and radiation oncologists. This type of research may help to design further perspective studies of differences in patient perceptions of AS using a more standardized approach to explain the concept, for example, a video or thirdparty explanation of what AS entails, as well as possible risks and benefits. However, the study originally was designed to explore factors influencing treatment decisions among men with LPC of all risk levels, not specifically men with low-risk disease. In addition, our study sample is younger (mean age, 58 years) than those reported previously, ${ }^{42,54,55}$ which may explain few of our men choosing WW/AS. Other limitations include the qualitative sample with possible selection bias and unknown recall bias. Also, we do not know if the physicians of study participants had a consistent understanding and use of the terms watchful waiting and active surveillance.

\section{Conclusions}

Most men considered WW/AS a risk they were not willing to take. The desire for aggressive therapy may reflect the complex psychology associated with receiving a diagnosis of cancer and the limited supportive counseling received, together with misunderstanding of both the risk of progression for low-risk disease and the survival benefits that aggressive treatment may provide. Lack of physician support is a significant barrier for men who were receptive to the WW/AS approach. Further education of physicians and patients and their families is essential to help men select an appropriate treat- 
ment that maximizes both survival and quality of life. Consensus still is needed on the definitions and use of WW and AS. Evidence is needed to identify the best candidates for AS and to standardize the AS protocol. ${ }^{41}$

The authors thank Dr. Joe Liu for his important suggestions on the manuscript revisions and Drs. Jeffrey Forman, Samuel Rosemberg, Tewodros Fesseha, Willie Underwood, III, and Issac Powell for referring patients for this study.

\section{References}

1. Welch HG, Albertsen PC. Prostate cancer diagnosis and treatment after the introduction of prostatespecific antigen screening: 1986-2005. J Natl Cancer Inst 2009;101:1325-9.

2. Welch HG, Black WC. Overdiagnosis in cancer. J Natl Cancer Inst 2010;102:605-13.

3. Roemeling S, Roobol MJ, de Vries SH, et al. Active surveillance for prostate cancers detected in three subsequent rounds of a screening trial: characteristics. PSA doubling times, and outcome. Eur Urol 2007;51:1244-51.

4. Draisma G, Boer R, Otto SJ, et al. Lead times and overdetection due to prostate-specific antigen screening: estimates from the European Randomized Study of Screening for Prostate Cancer. J Natl Cancer Inst 2003;95:868-78.

5. Draisma G, Etzioni R, Tsodikov A, et al. Lead time and overdiagnosis in prostate-specific antigen screening: importance of methods and context. J Natl Cancer Inst 2009;101:374-83.

6. Heijnsdijk EA, der Kinderen A, Wever EM, et al. Overdetection, overtreatment and costs in prostatespecific antigen screening for prostate cancer. $\mathrm{Br} \mathrm{J}$ Cancer 2009; 101:1833-8.

7. Mohler J, Bahnson RR, Boston B, et al. NCCN clinical practice guidelines in oncology: prostate cancer. J Natl Compr Canc Netw 2010;8:162-200.

8. Johansson JE, Andren O, Andersson SO, et al. Natural history of early, localized prostate cancer. JAMA 2004;291:2713-9.

9. Klotz L, Zhang L, Lam A, et al. Clinical results of long-term follow-up of a large, active surveillance cohort with localized prostate cancer. J Clin Oncol 2010;28:126-31.

10. Stattin P, Holmberg E, Johansson JE, et al. Outcomes in localized prostate cancer: National Prostate Cancer Register of Sweden follow-up study. J Natl Cancer Inst 2010;102:950-8.

11. Lu-Yao GL, Albertsen PC, Moore DF, et al. Outcomes of localized prostate cancer following conservative management. JAMA 2009;302:1202-9.

12. Harlan SR, Cooperberg MR, Elkin EP, et al. Time trends and characteristics of men choosing watchful waiting for initial treatment of localized prostate cancer: results from CaPSURE. J Urol 2003;170: 1804-7.

13. Miller DC, Gruber SB, Hollenbeck BK, et al. Incidence of initial local therapy among men with lowerrisk prostate cancer in the United States. J Natl Cancer Inst 2006;98:1134-41.

14. Cooperberg MR, Broering JM, Carroll PR. Time trends and local variation in primary treatment of localized prostate cancer. J Clin Oncol 2010;28: 1117-23.

15. Dall'Era MA, Cooperberg MR, Chan JM, et al. Active surveillance for early stage prostate cancer: review of the current literature. Cancer 2008;112: $1650-9$

16. Klotz L. Active surveillance for prostate cancer: for whom? J Clin Oncol 2005;23:8165-9.

17. Allaf ME, Carter HB. Update on watchful waiting for prostate cancer. Curr Opin Urol 2004;14:171-5.

18. Bailey DE Jr, Wallace M. Critical review: is watchful waiting a viable management option for older men with prostate cancer? Am J Mens Health 2007;1: $18-28$.

19. Steinberg GD, Bales GT, Brendler CB. An analysis of watchful waiting for clinically localized prostate cancer. J Urol 1998;159:1431-6.

20. Parker C. Active surveillance: towards a new paradigm in the management of early prostate cancer. Lancet Oncol 2004;5:101-6.

21. Thompson I, Thrasher JB, Aus G, et al. Guideline for the management of clinically localized prostate cancer: 2007 update. J Urol 2007;177:2106-31.

22. Chapple A, Ziebland S, Herxheimer A, et al. Is "watchful waiting" a real choice for men with prostate cancer? A qualitative study. BJU Int 2002;90: 257-64.

23. Holmboe ES, Concato J. Treatment decisions for localized prostate cancer: asking men what's important. J Gen Intern Med 2000;15:694-701.

24. Schwartz K, Powell IJ, Underwood W 3rd, et al. Interplay of race, socioeconomic status, and treatment on survival of patients with prostate cancer. Urology 2009;74:1296-302.

25. Shavers VL, Brown ML, Potosky AL, et al. Race/ ethnicity and the receipt of watchful waiting for the initial management of prostate cancer. J Gen Intern Med 2004;19:146-55.

26. Harlan LC, Potosky A, Gilliland FD, et al. Factors associated with initial therapy for clinically localized prostate cancer: prostate cancer outcomes study. J Natl Cancer Inst 2001;93:1864-71.

27. Krupski TL, Kwan L, Afifi AA, Litwin MS. Geographic and socioeconomic variation in the treatment of prostate cancer. J Clin Oncol 2005;23: 7881-8

28. Steenland K, Goodman M, Liff J, et al. The effect of race and rural residence on prostate cancer treatment choice among men in Georgia. Urology 2011;77: 581-7. 
29. Zeliadt SB, Ramsey SD, Penson DF, et al. Why do men choose one treatment over another?: a review of patient decision making for localized prostate cancer. Cancer 2006;106:1865-74.

30. Steenland K, Goodman M, Liff J, et al. Quality of life among men with prostate cancer in rural Georgia. Urology 2011;77:927-33.

31. Katz SJ, Lantz PM, Janz NK, et al. Patient involvement in surgery treatment decisions for breast cancer. J Clin Oncol 2005;23:5526-33.

32. Hu JC, Kwan L, Krupski TL, et al. Determinants of treatment regret in low-income, uninsured men with prostate cancer. Urology 2008;72:1274-9.

33. Xu J, Dailey RK, Eggly S, et al. Men's perspectives on selecting their prostate cancer treatment. JNMA 2011;103:468-78.

34. Rosenstock IM, Strecher VJ, Becker MH. Social learning theory and the Health Belief Model. Health Educ Q 1988;15:175-83.

35. Miller SM, Diefenbach MA. The Cognitive-Social Health Information Processing (C-SHIP) model: a theoretical framework for research in behavioral oncology. In Krantz DS, Baum A (eds). Technology and Methods in Behavioral Medicine. Mahwah, NJ: Lawrence Erlbaum; 1998:219-44.

36. Miller WL, Crabtree BF. Clinical research: a multimethod typology and qualitative roadmap. In Crabtree BF, Miller WL (eds). Doing qualitative research. 2nd edition. Thousand Oaks: Sage Publications; 1999;3-30.

37. Strauss A CJ. Basics of qualitative research: grounded theory procedures and techniques. Newbury Park: Sage Publications; 1990.

38. Lin K, Croswell JM, Koenig H, Lam C, Maltz A. Prostate-specific antigen-based screening for prostate cancer: an evidence update for the U.S. Preventive Services Task Force. Evidence synthesis number 90. AHRQ Publication No. 12-05160EF-1. October 2011. Available at http://www.us preventiveservicestaskforce.org/uspstf12/prostate/ prcascres.pdf. Accessed September 11, 2012.

39. Harris G. U.S. panel says no to prostate screening for healthy men [press release online]. New York Times. October 6, 2011. Available at http://www. nytimes.com/2011/10/07/health/07 prostate.html. Accessed October 142011.

40. Carroll PR. Early stage prostate cancer-do we have a problem with over-detection, overtreatment or both? J Urol 2005;173:1061-2.

41. National Institutes of Health State-of-the-Science Conference Statement. The role of active surveillance in the management of men with localized prostate cancer. Available at http://consensus.nih.gov/ 2011/docs/prostate/ASPC\%20Final\%20Draft\%20 Statement.pdf. Accessed January 6, 2012.
42. O'Rourke ME. Narrowing the options: the process of deciding on prostate cancer treatment. Cancer Invest 1999;17:349-59.

43. Davison BJ, Oliffe JL, Pickles T, Mroz L. Factors influencing men undertaking active surveillance for the management of low-risk prostate cancer. Oncol Nurs Forum 2009;36:89-96.

44. Gorin MA, Soloway CT, Eldefrawy A, Soloway MS. Factors that influence patient enrollment in active surveillance for low-risk prostate cancer. Urology 2011;77:588-91.

45. Demark-Wahnefried W, Schildkraut JM, Iselin CE, et al. Treatment options, selection, and satisfaction among African American and white men with prostate carcinoma in North Carolina. Cancer 1998;83: 320-30.

46. Moul JW, Douglas TH, McCarthy WF, McLeod DG. Black race is an adverse prognostic factor for prostate cancer recurrence following radical prostatectomy in an equal access health care setting. J Urol 1996;155:1667-73.

47. Amling CL, Riffenburgh RH, Sun L, et al. Pathologic variables and recurrence rates as related to obesity and race in men with prostate cancer undergoing radical prostatectomy. J Clin Oncol 2004;22: $439-45$.

48. Freedland SJ, Jalkut M, Dorey F, et al. Race is not an independent predictor of biochemical recurrence after radical prostatectomy in an equal access medical center. Urology 2000;56:87-91.

49. Resnick MJ, Canter DJ, Guzzo TJ, et al. Does race affect postoperative outcomes in patients with lowrisk prostate cancer who undergo radical prostatectomy? Urology 2009;73:620-3.

50. McVey GP, McPhail S, Fowler S, et al. Initial management of low-risk localized prostate cancer in the UK: analysis of the British Association of Urological Surgeons Cancer Registry. BJU Int 2010;106: 1161-4.

51. Payer L. Medicine and culture: varieties of treatment in the United States, England, West Germany, and France. New York: Henry Holt and Company; 1996.

52. National Cancer Institute. National Cancer Act of 1971. Available at http://dtp.nci.nih.gov/timeline/ noflash/milestones/M4 Nixon.htm. Accessed November 12, 2011.

53. American Cancer Society. Cancer facts \& figures 2011. Available at http://www.cancer.org/Research/ CancerFactsFigures/CancerFactsFigures/cancer-factsfigures-2011. Accessed December 11, 2011.

54. Denberg TD, Melhado TV, Steiner JF. Patient treatment preferences in localized prostate carcinoma: The influence of emotion, misconception, and anecdote. Cancer 2006;107:620-30.

55. Berry DL, Ellis WJ, Woods NF, et al. Treatment decision-making by men with localized prostate cancer: the influence of personal factors. Urol Oncol 2003;21:93-100. 